\title{
Fractional Quantum Chemistry
}

\author{
Mohammad Mostafanejad ${ }^{1}$ \\ ${ }^{1}$ Virginia Polytechnic Institute and State University
}

April 16, 2021

\begin{abstract}
The realization of fractional quantum chemistry is presented. Adopting the integro-differential operators of the calculus of arbitrary-order, we develop a general framework for the description of quantum nonlocal effects in the complex electronic environments. After a brief overview of the historical and fundamental aspects of the calculus of arbitrary-order, various classes of fractional Schrödinger equations are discussed and pertinent controversies and open problems around their applications to model systems are detailed. We provide a unified approach toward fractional generalization of the quantum chemical models such as Hartree-Fock and Kohn-Sham density functional theory and develop fractional variants of the fundamental molecular integrals and correlation energy. Furthermore, we offer various strategies for modeling static and dynamic quantum nonlocal effects through constant- and variable-order fractional operators, respectively. Possible directions for future developments of fractional quantum chemistry are also outlined.
\end{abstract}

\section{Hosted file}

main.pdf available at https://authorea.com/users/408104/articles/518255-fractional-quantumchemistry 
figures/power/power-eps-converted-to.pdf 